\title{
Characterisation and Processing of Reinforced PA 6 with Halloysite Nanotubes (HNT) for Injection Molding
}

\author{
Angel Fernández ${ }^{1,2, *}$-Manuel Muniesa ${ }^{1}$ - Jaime González² \\ 1 University de Zaragoza, Mechanical Engineering Department, Spain \\ 2 Fundación AITIIP, Parque Tecnológico Cogullada, Spain
}

The use of nano-scaled reinforcements enchances the mechanical performance of the resulting material and the addition of natural origin nanotubes improve the sustainability and environmental impact of the product. The present work describes the preparation, characterization and processing of nanocomposites based on thermoplastic Polyamide 6 and Halloysite nanotubes (HNT).

The nanocomposites were prepared in several stages and the results ensure the final application at "industrial scale". The first formulation was a high concentration of Halloysite nanotubes (up to 30\% weight content) made with a twin screw extrusion compounder which was used as a masterbatch. Application materials were obtained reducing the masterbatch in raw PA 6 down to 3 or $6 \%$ of HNTs weight content. In this stage the influence of use or not an extruder-compounder was analyzed. Finally, a low cost preparation and sustainable development technique as injection moulding was applied to obtain test parts of all developed materials.

Results were analyzed at a microscopic scale (TGA, FTIR, XRD, SEM, TEM) and macroscopic scale (rheology, mechanical properties and flame resistance). TGA and FTIR results show that the content of HNTS was as accurate as expected. XRD results showed a greater interaction between the HNTS and the matrix if the extrusion-compounding process was used before injection moulding. SEM and TEM results showed a better dispersion of HNTS if extrusion was performed at high screw rotation speed. All analysis concluded the nanocomposites can be processed on standard equipment due to the resultant low viscosity. Also, low content of HNTs proved that mechanical properties are highly improved as flame resistance remains equivalent.

Keywords: nanocomposites, Halloysite nanotubes, injection moulding, compounding

\section{O INTRODUCTION}

The newest requirements for lightweight automotive materials embrace improved security performance (high impact energy absortion), lower weight (equivalent mechanical properties with lower density) and improved functional specifications (higher flame retardancy and restricted volatile emission). The new hybrid and electric vehicles (HEV) require the key factors indicated to reduce the weight of existing parts to accommodate and new components as the battery pack; all with at least the same security and the greatest possible autonomy.

Nanocomposites emerge as an alternative to existing materials to meet these new requirements. For this reason the research described in this paper focuses on the characterization and processing at the industry scale of nanocomposites based in thermoplastic PA 6 polymeric matrix and Halloysite nanotubes (HNT) as nano-scaled reinforcement. The polyamide 12 nanocomposites based on halloysite nanotubes are promising candidates for structural applications as Lecouvet [1] studied with a mini-compounder.

The use of nano reinforcements extremely increases the interaction between the resin and the charge resulting in stronger and lighter materials with special properties. The use of Halloysite nanotubes improves the mechanical properties of polyamide using a lower amount of charge if compared with long fiber glass or glass micro spheres so that same properties are affordable with lower densities.

Nanocomposites made of polyamide and Halloysite nanotubes are of interest because of the natural origin of these nanotubes. These nanotubes not only give good mechanical properties but also low price and sustainability. The Naturalnano Company [2] offers commercial grades of nanocomposites with PP, PA 6 or EPDM matrix. Ulrich's [3] research described the influence of the molecular weight on thermal, mechanical and rheological properties concluding HNTs were usable and an alternative to Carbon nanotubes (CNT) based nanocomposites because their cost competitiveness.

The methodology to prepare PA/HNT nanocomposites requires the functionalization of nanotubes using amines or block copolymers to improve interfacial interactions with the matrix polymer as studied by Deliang [4]. Previously, the purity of HNT must be the maximum. Functionalized nanotubes have to be solved for different raw materials. Optimization of this process implies the suppression of agglomerates and aggregates of nanotubes as their exfoliation to improve the maximum contact with polymer getting a real nanocomposite. Previous developments are not limited to polyamide matrix nanocomposites (e.g. PA 6). Also PP and EPDM matrix have been studied in detail by Ismail [5] and Paspakhsh [6]. The application of their results has led 
to new and commercial products as Pleximer-PP and Nubrid respectively in addition to cited Pleximer-PA [2].

As all nanocomposites based on thermoplastic matrices are challenged to be made in the form of pellets by low cost preparation process like extrusioncompounding and for subsequent use by injection molding, this research used these two technologies. The novel Polyamide based nanocomposites are well described by Hedicke-Hochstotter [7] as a ready-toprocess material. Obtaining nanocomposites readyto-inject through an extrusion-compounding machine in a one-step-process at industrial scale is extremely difficult with standard equipment. Previous research at lab. scale have demonstrated the need to optimize the extrusion process to disperse enough the nanotubes combined with recirculation process to increase the residence time in order to ensure the correct diffusion of the matrix. Only if the nanotubes are dispersed properly in the thermoplastic matrix, they will remit efficiently its properties to the resulting product.

The complexity of this process is such that it was decided to produce a masterbatch with high concentration of nanotubes with the extruder. So the object of the research was to use the masterbatch to prepare different nanocomposites reducing the weight content of nanotubes. Also, it was studied the feasibility of dispersing masterbatch directly in the injection machine. All extrusion and injection moulding equipments used were industrial-scaled size.

Physicochemical analysis has combined nano and micro techniques as X-ray dispersion (XRD), Fourier transform infrared spectrometry (FTIR), Scanning electron microscopy (SEM) and Transmission electron microscopy (TEM). XRD analysis is focused to previous studies where Baochun [8] and [9] discovered the effect of the halloysite in the crystallization of polyamide. Those revealed that with more interaction acceleration of crystallization and the increment of $\gamma$ phase occurs. This $\gamma$ phase appeared against the $\alpha$ phases usually present in PA 6. This research was demonstrated with mini-lab extruders.

Improvements in mechanical behaviour regarding the essential work of fracture (EWF) at low contents of halloysite nanotubes has been studied by Prashantha [10] with additional positive conclusions about the increase in storage modulus, young modulus, tensile strength and notched Charpy impact strength without loss of ductility at laboratory scale.

The fire behaviour of PA $6+$ HNT composites was studied by Marney [11] with positive conclusions about the ease of composite preparation as an attractive consideration for further development or study of the systems of flame inhibition of PA 6 .

The main goal of this research is to study the effects on the distribution and dispersion of HNT in PA 6 prepared by extrusion and injection molding, as well as to measure the viscosity, stiffness and flame retardancy of the obtained nanocomposites Furthermore, extrusion and injection moulding have been applied with industry scaled equipment. The conclusions of this research have answered the uncertainities of key factors affecting the design for manufacturing of newer parts made in nanocomposites where feasibility and performance are so close that they fit the newest product requirements.

\section{EXPERIMENTAL}

\subsection{Materials}

Three materials were used: Raw PA 6 Badadur from BADA Hispanaplast, raw milled Halloysite nanotubes from Naturalnano, Inc, and Pleximer-PA, a masterbatch of PA 6 up to $30 \%$ weight content of Halloysite nanotubes also from Naturalnano.

\subsection{Processing Equipment}

A Coperion ZSK 26 extrusion-compounding machine equipped with $29 \mathrm{~mm}$ diameter twin-screw and two gravimetric feeders was used for masterbatch preparation. This machine was used also to reduce the content of nanotubes in different blends.

A JSW 85 EL II electrical injection moulding machine was used to inject test specimens in a specific mold.

\subsection{Testing Equipment}

A Gottfert 1500 capillary rheometer was used for rheometry and an Instrom 8032 test machine was used for tensile and flexural test.

For physicochemical analysis the XRD (Rygaku/ Max System) was used to study the crystalline structure of materials. The TGA (Mettler Toledo TGA/STDA $851 \mathrm{SF} / 1100$ ) was used to measure the variation of properties with temperature in a range from 30 to $800{ }^{\circ} \mathrm{C}$ using. Finally, the FTIR (Bruker Vertex 70 with ATR Golden Gate accessories) was used to analyze the chemical composition of final materials as well as the surface atomic composition was determined using XPS (Kratos Tech Axis Ultra DLD) using AlK $\alpha$ radiation at $15 \mathrm{kV}$ and $10 \mathrm{~mA}$. 
The morphologies of nanocomposites were observed on cryofractured surfaces using a S2300 (Hitachi) scanning electron microscope. SEM equipment works within a range from 0.5 to $30 \mathrm{kV}$. Specimens were covered with a thin gold layer.

A transmission electron microscope 2000-FXII (JEOL Ltd) with $200 \mathrm{kV}$ acceleration voltage was used to study the dispersion of HNT inside the polyamide matrix. The TEM specimens were cut in ultrathin sections of approximately $100 \mathrm{~nm}$ with a microtome, collected on copper grids and covered with a carbon thin layer.

\subsection{Methodology}

The methodology included the following stages:

- A masterbatch of raw PA 6 with a maximum weight content of $30 \%$ of Halloysite nanotubes was made by extrusion-compounding at barrel temperature of $250{ }^{\circ} \mathrm{C}$ and screw speed of 450 rpm. After several trials the results were not as good as expected with several interruptions because power stucked the hopper wall blocking the inlet of the extruder. Due to the difficulties of reliability of the trial the alternative chosen option was to buy the masterbatch containing $30 \%$ wt HNT (Pleximer-PA supplied by Naturalnano, INC.). The specifications of HNTs in the masterbatch were in a range of diameter 8 to $10 \mathrm{~nm}$, mean length of nanotubes $1.5 \mu \mathrm{m}$, and a purity of $95 \%$.

- Different blends of PA 6/HNTs were made reducing the masterbatch into Raw PA 6 by extrusion-compounding at a barrel temperature of $250^{\circ} \mathrm{C}$ and two different screw speeds of 450 and $900 \mathrm{rpm}$ for a constant output of $20 \mathrm{~kg} / \mathrm{h}$. The weight content of HNTs was selected to 3 or $6 \%$ programming the gravimetric feeder.

- After pelletizing, the nanocomposite granules, the raw PA 6 and the masterbatch were tested in a capillary rheometer at temperatures of 240 to 260 ${ }^{\circ} \mathrm{C}$ and a $1 \mathrm{~mm}$ diameter capillar.

- For mechanical testing, nanocomposite granules, raw PA 6 and masterbatch were injection-molded into standart test specimen for tensile and flame retardancy test (Fig. 1). The processing conditions were; Barrel temperature profile 245, 245 and $255^{\circ} \mathrm{C}$ (optimized from the hopper to the nozzle); nozzle temperature $255^{\circ} \mathrm{C}$; screw rotating speed $70 \mathrm{rpm}$ (for a screw diameter of $36 \mathrm{~mm}$ ); back pressure $4.5 \mathrm{MPa}$; mould temperature $70{ }^{\circ} \mathrm{C}$ (hot water); filling time $1.2 \mathrm{~s}$; injection speed linearly profiled from 20 to $40 \%$; maximum injection pressure (down $35 \mathrm{MPa}$ for all tests); holding pressure $75 \mathrm{MPa}$; hold pressure time $8.5 \mathrm{~s}$; cooling time $22 \mathrm{~s}$ and total cycle time $40 \mathrm{~s}$.

- For physicochemical characterization small pieces of granules and injected parts were analyzed by XRD, TGA, FTIR and XPS.

- For morphological characterization SEM and TEM microscopic images were taken on cryomicrotomic laminates extracted from the surfaces of the nanocomposite.

- For mechanical characterization all compounded PA 6 based materials were moisture conditioned at $25{ }^{\circ} \mathrm{C}$ room temperature and $50 \%$ ambient humidity. Flexural modulus calculation was made accordingly to ISO-178 for 3 point bending (thickness-span ratio of $1: 16$ and cross head displacement rate of $2 \mathrm{~mm} / \mathrm{min}$ ). The dimensions of bending specimens were $80 \times 10 \times 4 \mathrm{~mm}$. Tensile modulus and strength calculation was made accordingly to ASTM D638-72 (crosshead rate of $20 \mathrm{~mm} / \mathrm{min}$ ). These parameters could be directed obtained from $\sigma-\varepsilon$ curves.

- Flame resistance evolution was made accordingly to Underwritters Laboratories (UL94-V0, V1 and V2) test for vertical combustion with Bunser flame.

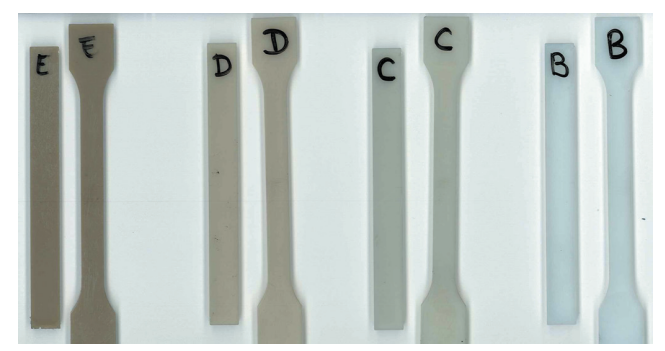

Fig. 1. Injected parts (E-PA 6+30 \%wt HNT, B-rawPA 6, C-PA 6+3 \%wt HNT, D-PA 6+6 \%wt HNT)

\section{EXTRUSION-COMPOUNDING AND INJECTION MOULDING TESTS}

The twin-screw extrusion-compounder was used to prepare different blends of PA 6 and reinforced masterbatch. The pellets obtained were analyzed or injected afterwards. Selected cases are summarized in Table 1. Case R is raw PA 6 and Case M is a Masterbatch of PA 6 with $30 \%$ of HNT. Cases E1 to $\mathrm{E} 4$ are blends of different proportions of $\mathrm{R}$ and $\mathrm{M}$ pellets to achieve new nanocomposites reducing the content of HNT to 3\% (E1 and E2) and 6\% (E3 and E4). The variation of extrusion speed was also studied. Cases E1 and E2 were performed at high extrusion speed (900 rpm) as E3 and E4 were made slower (450 
rpm). All cases have been characterized at micro and nano scale.

The injection moulding machine was used to inject test specimens using extrusion-compounded materials and others. All these test parts were injected under optimum processing conditions regarding the knowledge of the Technological Centre about injection of reinforced PA 6 and their own methodology to set up the process. Selected cases for analysis and discussion are RI, MI, E2I, E4I, DI1 and DI2. Cases RI, MI, E2I and E4I are injected samples of R, M, E2 and E4 materials. The influence of using the extrusion process to disperse the HNT through the polymer was studied applying Direct Injection (DIi) and comparing both results. DI1 and DI2 are injected samples of manual mixtures of $\mathrm{R}$ and $\mathrm{M}$ pellets. Their weight content of HNT is 3 and $6 \%$ respectively. All these cases were repeated 10 times after enough neglected cycles waiting for a stabilization of process conditions in the case of Injection molding. All injected specimens have been characterized at macro, micro and nano scale.

Table 1. Extrusion-Compounded materials

\begin{tabular}{clcc}
\hline Case No & Resultant composition & $\begin{array}{c}\text { Extr. speed } \\
{[\mathrm{rpm}]}\end{array}$ & $\begin{array}{c}\mathrm{R} / \mathrm{M} \text { blend } \\
{[\%]}\end{array}$ \\
\hline $\mathrm{R}$ & PA 6 & - & $100 / 0$ \\
\hline M & PA 6+30 \%wt HNT & - & $0 / 100$ \\
\hline E1 & PA 6+3 \%wt HNT & 900 & $90 / 10$ \\
\hline E2 & PA 6+3 \%wt HNT & 450 & $90 / 10$ \\
\hline E3 & PA 6+6 \%wt HNT & 900 & $80 / 20$ \\
\hline E4 & PA 6+6 \%wt HNT & 450 & $80 / 20$ \\
\hline
\end{tabular}

\section{RESULTS AND DISCUSSION}

Different results have been obtained from the analysis of the materials and test parts. These results describe the characterization of the nanocomposites from different points of view: the rheological behaviour, the mechanical properties, the homogeneity of the nanocomposite and the interaction between matrix and filler and the stiffness of the product. Therefore, these results are analyzed at a different size scale depending on the nature of the test.

\subsection{Physicochemical Characterization}

The results of Fourier transform infrared spectroscopy (FTIR), Thermogravimetric analysis (TGA) and X-ray diffraction (XRD) were obtained in order to discuss the chemical composition, degradation temperature and crystallographic structure of the nanocomposites after extrusion-compounding and injection molding.
The measurement of degradation temperature with TGA analysis showed the differences between different nanocomposites were neglectable and the values were $438{ }^{\circ} \mathrm{C}$ for all cases. This result was independent of HNTs weight content and processing conditions. Fig. 2 shows TGA results for E2I and E4I cases with a residual content similar to the halloysite weight content in the nanocomposite (3 to $6 \%$ respectively).

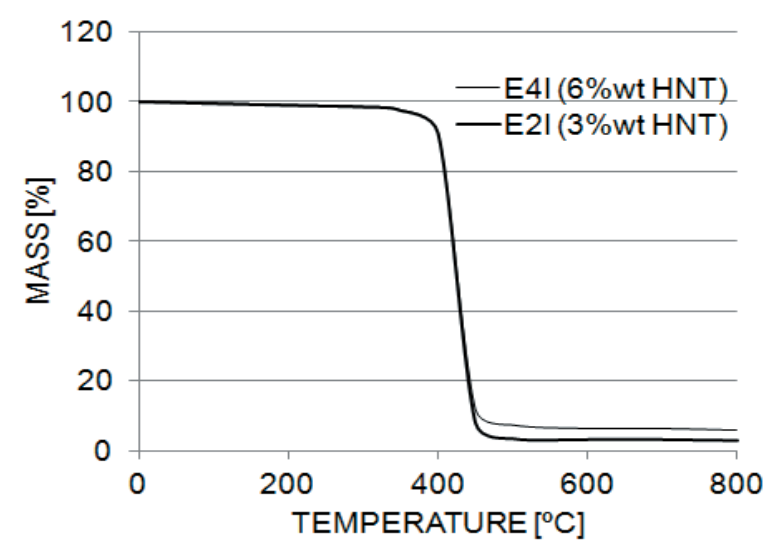

Fig. 2. TGA results for cases $E 2 I$ and $E 4 I$

The measurement of chemical composition with FTIR showed clear differences between different samples depending on the weight content of nanotubes. Fig. 3 shows FTIR results for materials M, E1 and E3. The curves are quite similar except in the influence area of the characteristic links of the groups present in the nanotubes. A detailed graphic of this area is shown in Fig. 4. At the way number associated to the stretchment of the links of Si (Si-O-Si: $1088 \mathrm{~cm}^{-1}$, Si-O-Al: $1032 \mathrm{~cm}^{-1}$ ) the curves grow proportionally to the content of nanotubes as expected so it can be concluded that the local presence of dispersed nanotubes has been achieved. Other relevant links are $\mathrm{Si}-\mathrm{O}-\mathrm{Si}$ (open chain siloxanes) at $940 \mathrm{~cm}^{-1}$.

The comparison of material curves with similar content of HNT like, E1, E2 and E2I and DI1 have shown that they are almost equal, so in this case, the results are independent of the extrusion speed (450 or $900 \mathrm{rpm}$ ) and the method of preparation of the material (extrusion or extrusion plus injection). The same conclusions can be drawn in comparisons between samples E3, E4, E4I and DI2.

The measurement of crystallographic structure with XRD has clarified the interaction between different phases and substances in the nanocomposites. In fact, XRD has been the analytical technique that has detected differences between all the materials and their way of preparation. 


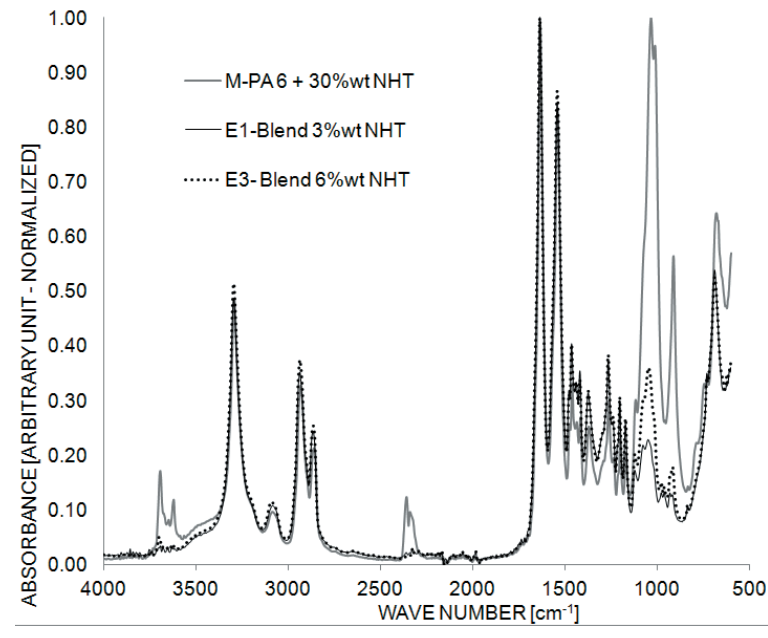

Fig. 3. FTIR results for Cases $M, E 1$ and $E 3$

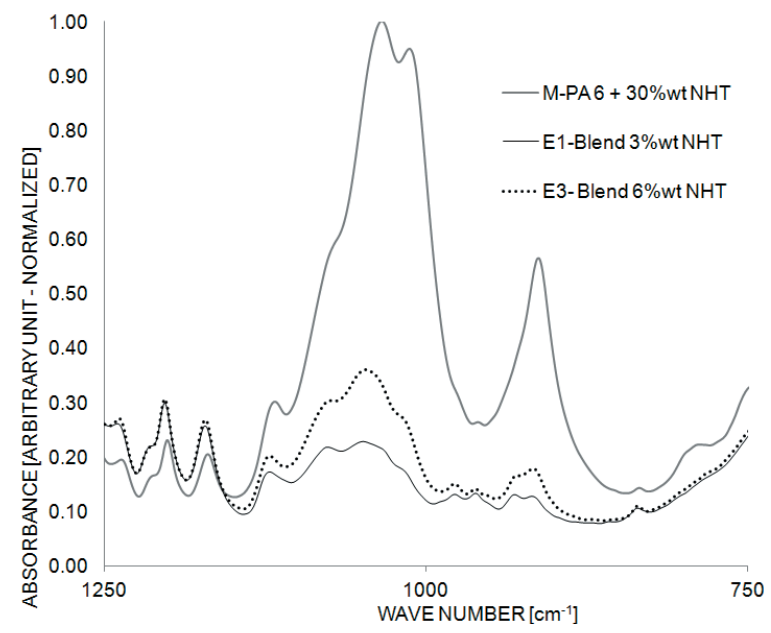

Fig. 4. Detailed FTIR for Cases $M, E 1$ and $E 3$

The results of all materials have been unified in Fig. 5. This graphic compares the WRD result for cases M, E1, E3I, DI1 and R combined also with the raw Halloysite nanotubes. In this graphic the dominant presence of $\alpha_{1}$ and $\alpha_{2}$ phases of raw PA 6 (R) can be easily dominant and, in the opposite site the dominant $\gamma$ phase (metastable) of the masterbacth (M) due to interaction a high halloysite-polymer that influences the acceleration of crystallinity growth and increases the presence of this phase.

Fig. 6 presents a detail of all the above mentioned cases to clarify the discussion. Raw PA 6 (R) exhibits $\alpha_{1}$ and $\alpha_{2}$ phases at $20.50^{\circ}$ and $22.82^{\circ}$ to $23.44^{\circ}$. On the other hand, high concentration HNTs masterbatch (M) exhibits $\gamma$ phase that appears at $21.26^{\circ}$. This result is as precise as expected in relation to previous research studies cited in the introduction. As HNT act as nucleating agent and accelerate the crystallization, the higher the HNT content is, the larger the presence of $\gamma$ phase crystal take up. The raw Halloysite curve exhibits a peak at $24.89^{\circ}$. Only a high content of Halloysite as case $M$ has shown the same peak, so that the rest of samples do not show the Halloysite characteristic peak due to their low content on nanotubes.

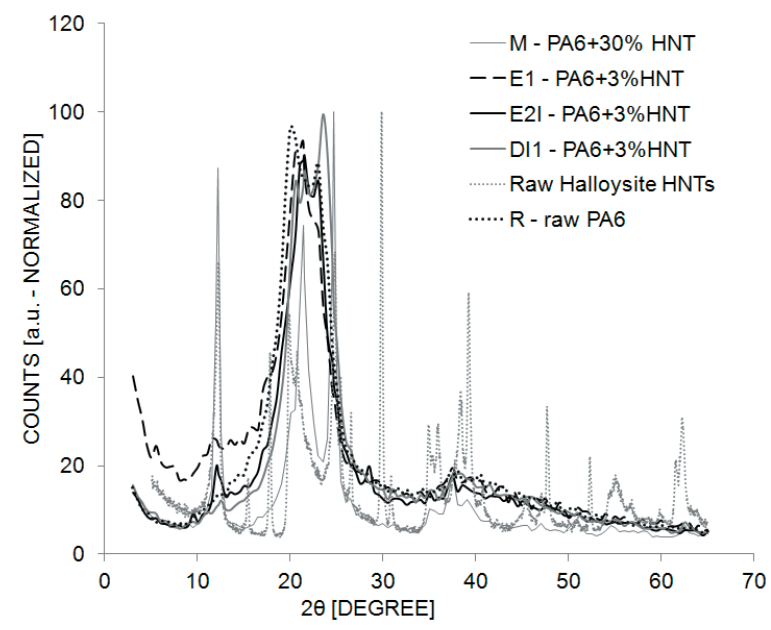

Fig. 5. XRD results for Cases $M, E 1, E 2 I, D I 1, R$ and raw Halloysite

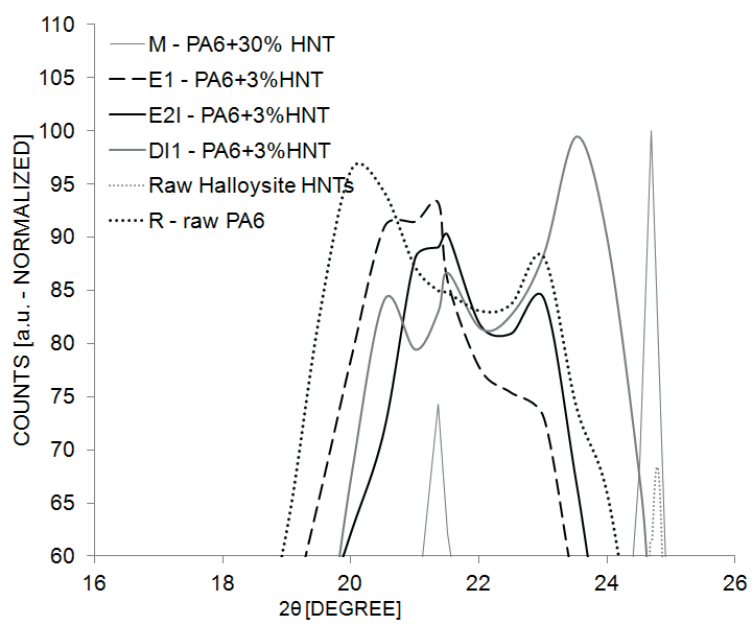

Fig. 6. XRD details for Cases $M, E 1, E 2 I, D I 1, R$ and raw Halloysite

The analysis of curves E1, E2I and DI1 should be very similar as they all PA $6+3 \%$ wt HNT with identical proportions of $\mathrm{R}$ and $\mathrm{M}$ polymers. However, their XRD curves show differences in polyamide phases distribution.

In the case of E1 material, the resultant curve shows the dominant phase is $\gamma$ with smaller "shoulders" that reveal a lower presence of $\alpha$ phases. The E1 material is made by extruding $\mathrm{R}$ and $\mathrm{M}$ materials at the highest twin-screw rotation speed of $900 \mathrm{rpm}$. It can therefore be concluded that a good dispersion of phase $\mathrm{M}$ has been dispersed in the whole 
resultant nanocomposite and the highest interaction between Halloysite nanotubes with the polymer has been achieved as this blend exhibits the maximum value of $\gamma$ phase.

The E2I material curve shows peaks of $\gamma$ and $\alpha_{2}$ phases to be almost equal. In this case, the material has followed two extrusion processes: The first in the compounding machine, and the second in the injection molding machine during the plastication stage. The injection molding process also substantially increased the residence time of the polymer at high temperature and it should have improved the correct dispersion of nanotubes if their separation and exfoliation were optimal. The main process difference between E1 and E2I is that during extrusion, E2I rotated at half speed than E1, so the friction was quite lower to result in a lack of nanotubes dispersion. This result proves the importance of extrusion speed as a mechanism of friction to ensure the good dispersion of additives including nano scaled reinforcements.

The worst result happened in the case the blend was mixed directly inside the injection machine hopper (DI1). In this case, the mixture of $\mathrm{R}$ and $\mathrm{M}$ was made manually and not mechanically. Then, $\alpha$ phases appeared roughly revealing a lack of interaction between polymer and reinforcement.

The results obtained in the case E2, E4I and DI2 which correspond to $6 \%$ weight content of HNT were similar to those presented for 3\% wt HNT.

\subsection{Rheological Characterisation}

The results of Capillar rheometry were obtained in order to discuss the rheological behaviour of the blends. The viscosity measurement and the viscous models generation are necessary for the discussion about the processability of the newer materials.

The measurement of apparent viscosity was made in a capillary rheometer for R, E2, E4 and M pellets. Three temperatures were pre- selected for each set measure, each of them embracing a large range of shear rates and viscosities. After the measurement the Rabinowitsch correction was applied and viscosity and shear stress were determined. These trials were repeated five times for each temperature. Figs. 7 to 9 show the viscosity versus shear rate and the tendency curve for each material at 240,250 and $260{ }^{\circ} \mathrm{C}$, respectively.

The results show that the viscosity dependency with shear rate of the nanocomposites in molten state is independent on the weight content of HNTs and remains similar to any thermoplastic. The analysis of the charts proves that the raw PA 6 (case R) is quite

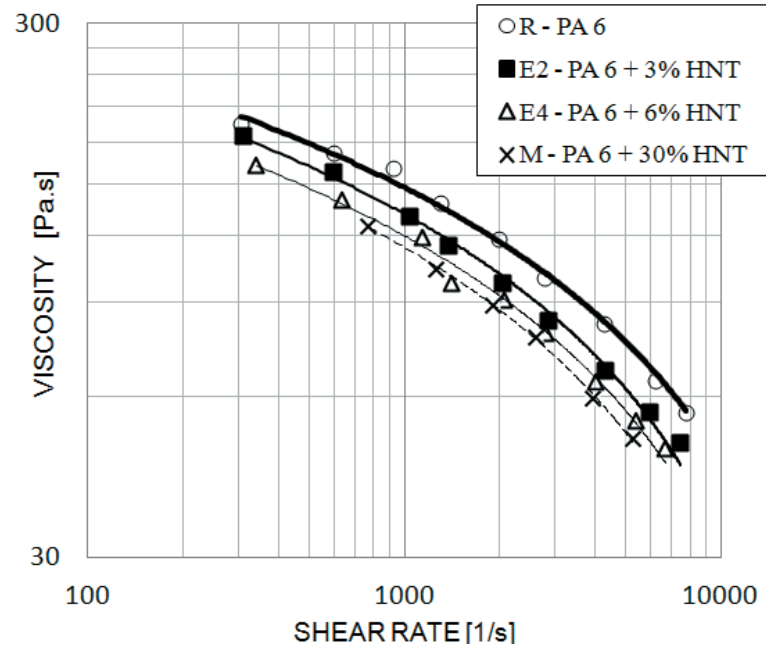

Fig. 7. Graphical comparison of measured viscosity and regression viscosity curves at $240^{\circ} \mathrm{C}$

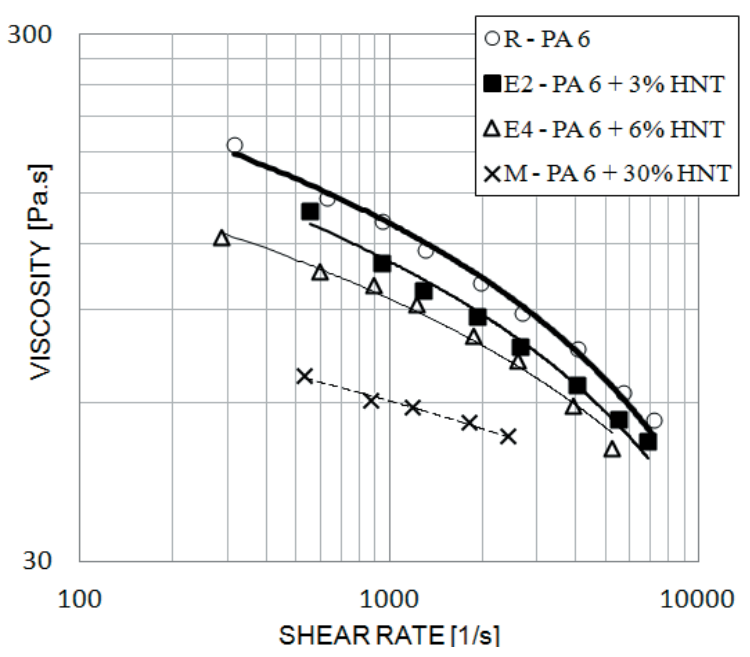

Fig. 8. Comparison of viscosities at $250^{\circ} \mathrm{C}$

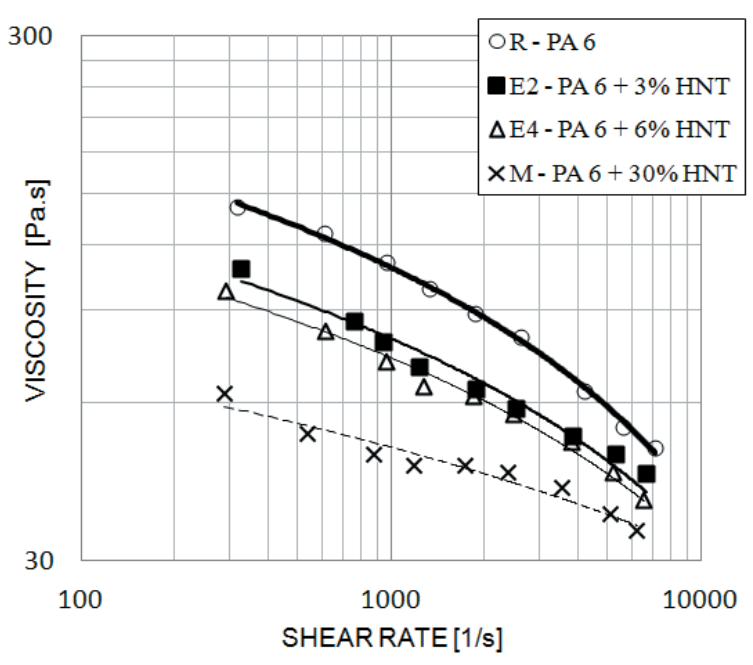

Fig. 9. Comparison of viscosities at $260^{\circ} \mathrm{C}$ 
more viscous than the masterbatch (case $\mathrm{M}$ ). For this reason, the blends of them $(\mathrm{R} / \mathrm{M})$ show intermediate values of viscosity, increasing as HNTs weight content is reduced. The reason is that rheological behaviour is highly influenced by the matrix which is a blend of different PA 6 grades.

The fitting of corrected viscosity data returns the coefficients for Carreau-WLF viscous model using the software Cadmould 3D-F SimFit v0.57. These are shown in Table 2. This model is necessary to perform injection moulding simulations with typical softwares like Moldflow, Cadmould 3D-F or Moldex and follows the following expression for the viscosity dependency on shear rate and temperature:

$$
\begin{gathered}
\eta=\frac{P_{1} \cdot a_{T}}{\left(1+\dot{\gamma} \cdot P_{2} \cdot a_{T}\right)^{P_{3}}}, \\
\log _{10}\left(a_{T}\right)=\frac{8.86\left(T_{0}-T_{s}\right)}{101.6+\left(T_{0}-T_{s}\right)}-\frac{8.86\left(T_{0}-T_{s}\right)}{101.6+\left(T_{0}-T_{s}\right)} .
\end{gathered}
$$

Table 2. Carreau-WLF model coefficients for R, M, E2 and E4 materials

\begin{tabular}{cccccc}
\hline CASE & $P_{1}[\mathrm{~Pa} \cdot \mathrm{S}]$ & $P_{2}[\mathrm{~s}]$ & $P_{3}[-]$ & $T_{0}\left[{ }^{\circ} \mathrm{C}\right]$ & $T_{S}\left[{ }^{0} \mathrm{C}\right]$ \\
\hline $\mathrm{R}$ & 203.0 & $1.11 \mathrm{e}^{-3}$ & 0.601 & 250 & 46.63 \\
\hline $\mathrm{M}$ & 81.26 & $1.89 \mathrm{e}^{-4}$ & 0.921 & 250 & 126.9 \\
\hline $\mathrm{E} 2$ & 163.9 & $1.19 \mathrm{e}^{-3}$ & 0.567 & 250 & 105.0 \\
\hline $\mathrm{E} 4$ & 136.8 & $6.94 \mathrm{e}^{-4}$ & 0.671 & 250 & 97.21 \\
\hline
\end{tabular}

The results shown in Figs. 7 to 9 have shown that the viscosity of nanocomposites does not increase dramatically with the content of nanotubes but it is within the order of magnitude of polymer matrix. The final values of viscosity are correlated to the portion of each component in the final blend. These intermediate values of viscosity could be explained by a good mixture of both materials ( $R$ and $M$ ) as mechanical and heat treatment is applied in the extruder without additional interaction between the nanotubes and the new matrix material in molten state. Furthermore, good dispersion could be achieved with good interaction of both polyamides.

The results shown in Table 2, after rheological characterization by fitting the measured values, show the feasibility of injection molding process for most applications and nearly any kind of parts with these materials as the viscosity is not so high.

The successful obtainment of the coefficients of Carreu-WLF model for all materials ensured that injection molding simulation could be performed for these materials. In the development of newer parts in the future, the use of process simulation is a critical stage for the final part development under feasibility criteria, mold design and manufacturing, and robust serial production.

\subsection{Mechanical Characterization}

The results of the tensile test are shown in Table 3 . They represent the averaged values for 10 different specimens for each material. Both elastic modulus and maximum stress increase with HNT content as expected. However, yield stress and elongation at break increase with HNT content up to 3 to $6 \%$, decreasing for higher nanotubes content.

Table 3. Results for mechanical properties of selected cases

\begin{tabular}{ccccc}
\hline CASE & $\begin{array}{c}\text { Elastic Modulus } \\
{[\mathrm{MPa}]}\end{array}$ & $\begin{array}{c}\text { Yield Stress } \\
{[\mathrm{MPa}]}\end{array}$ & $\begin{array}{c}\text { Max. Stress } \\
{[\mathrm{MPa}]}\end{array}$ & $\begin{array}{c}\text { Elong. at } \\
\text { break [\%] }\end{array}$ \\
\hline $\mathrm{R}$ & 2123 & 48.32 & 58.00 & 45.03 \\
\hline E21 & 2542 & 49.80 & 63.24 & 43.75 \\
\hline E4I & 3054 & 45.54 & 69.15 & 45.06 \\
\hline $\mathrm{M}$ & 3070 & 36.60 & 61.39 & 16.48 \\
\hline
\end{tabular}

The most important result is the loss of ductility for high HNTs concentration (30\%). The most appropriate explanation for the tendency of the evolution of mechanical properties lies in the process, so the specimens were fabricated as explained below. Cases R, E2I and E4I were previously extruded but masterbatch (M) was injected as it was supplied, without an additional extrusion process.

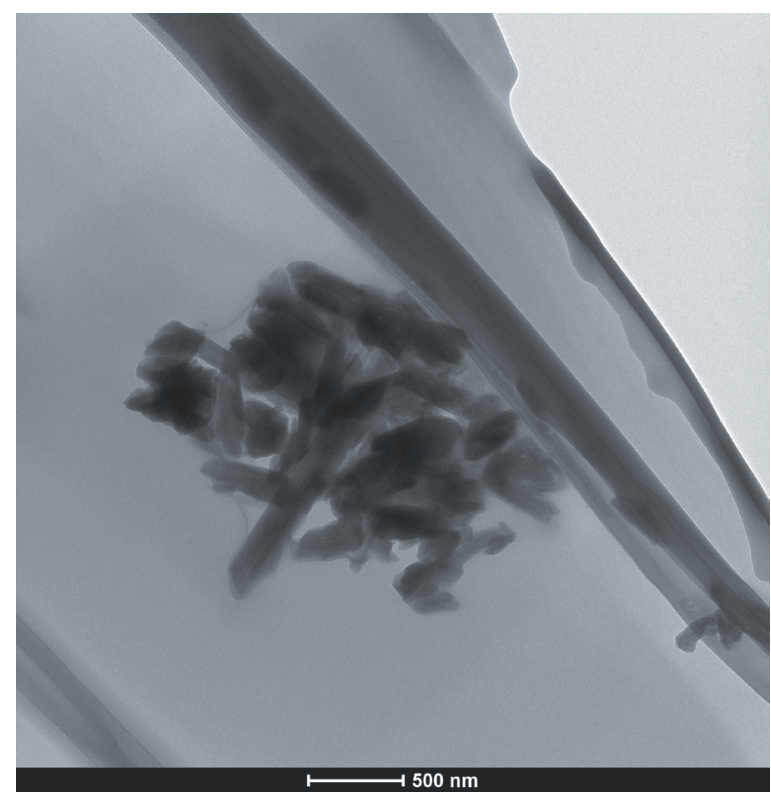

Fig. 10. Nanotubes aggregates 


\subsection{Morphology Characterization}

The results of SEM microscopy clarified the tensile test results. Fig. 10 shows the tendency of Halloysite nanotubes to create aggregates of $1 \mu \mathrm{m}$ minimum size. Analyzing the SEM images (Fig. 11) of the masterbatch (M) a lot of aggregates of nanotubes impossible to disperse in the matrix at nanometric scale can be seen. The low values for elongation at break for the masterbatch could be a result of these singularities in the material structure.

Aggregates were transferred to the molded part if there was no pre-extrusion process as shown in Fig. 11. In this case the direct mixing of $\mathrm{M}$ and $\mathrm{R}$ in the hopper did not prevent this problem.

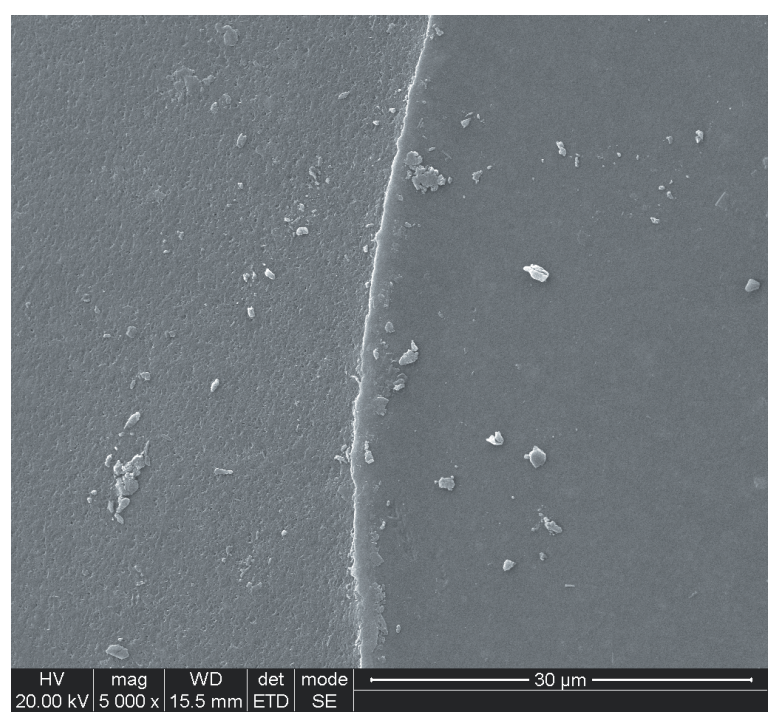

Fig. 11. SEM of $M$ with HNT aggregates

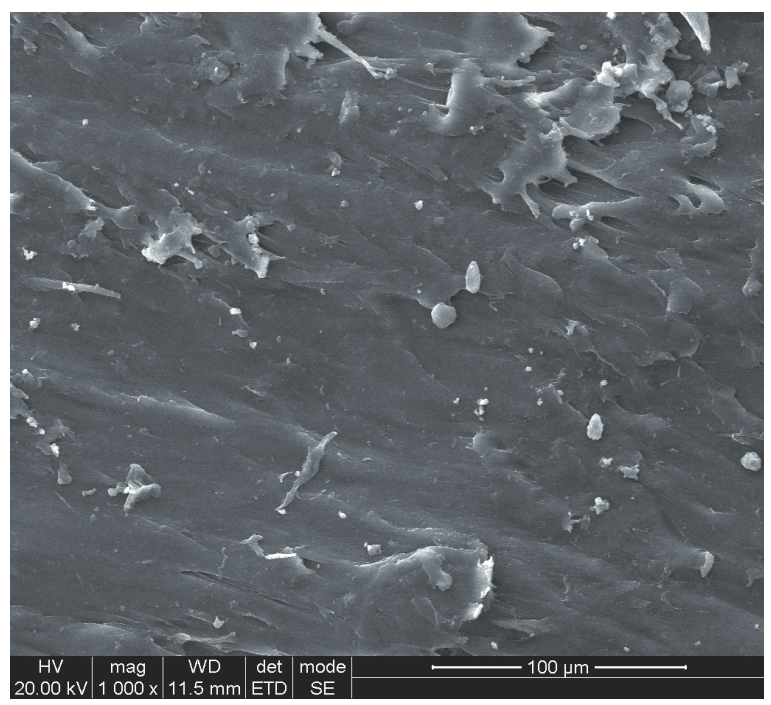

Fig. 12. SEM of D2I with HNT aggregates

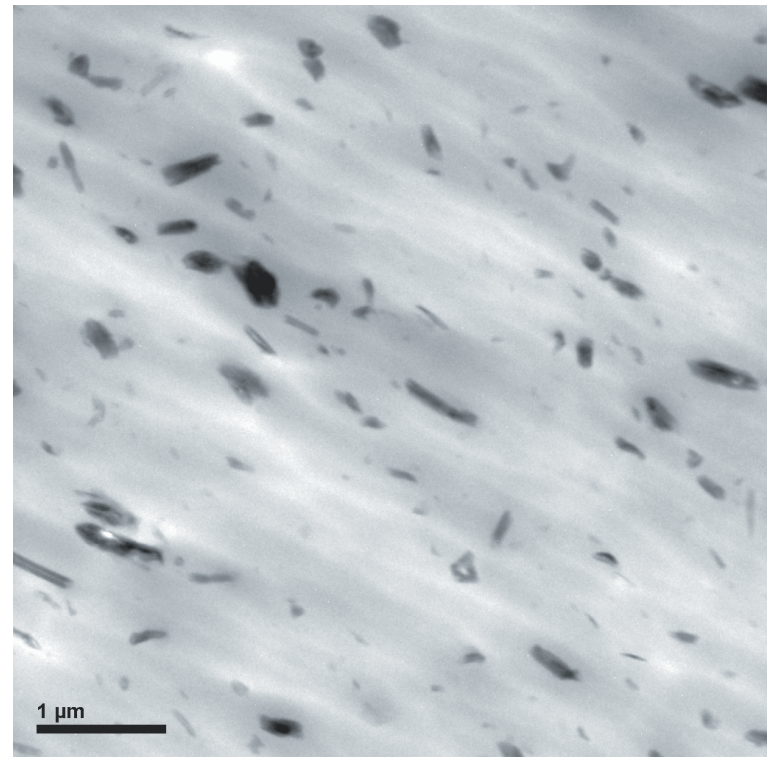

Fig. 13. TEM image of E2I case

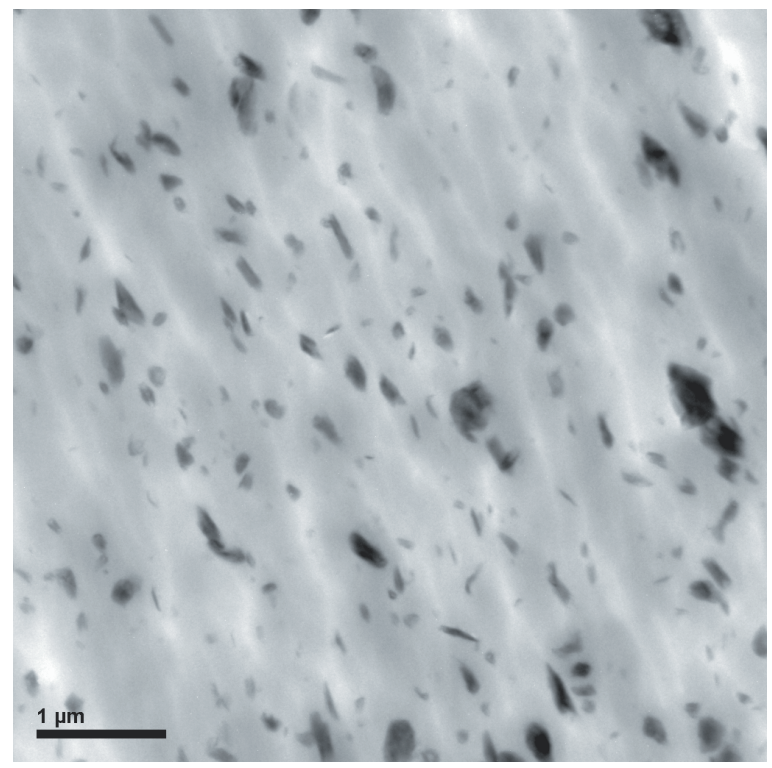

Fig. 14. TEM image of E4I case

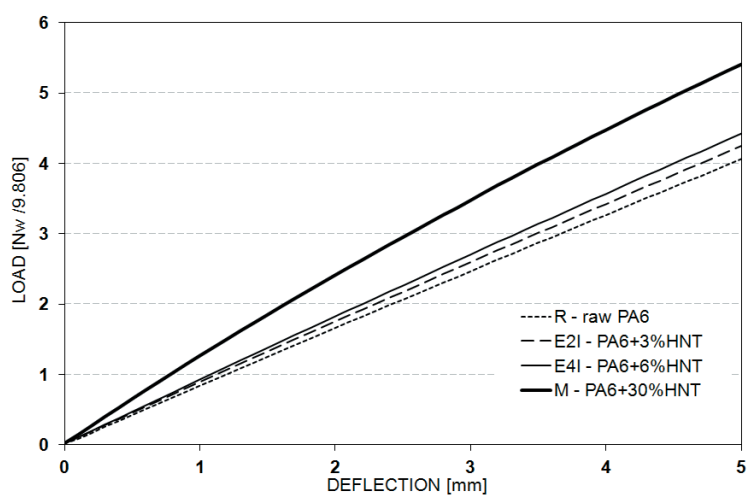

Fig. 15. Load-deflection results for bending test 
For this reason, previous extrusion process to injection molding improved the dispersion of nanotubes and resulted in better mechanical performance. Figs. 13 to 14 show the distribution of nanotubes in polymer matrix corresponding to cases E2I and E4I. Also, trials developed at higher rotation speeds of the extruder (E1I and E3I) resulted in better nanotubes dispersion.

\subsection{Product Stifness}

In order to evaluate the mechanical performance of the materials R, E2I, E4I and M, the bending stiffness of the test part was calculated as the ratio load-displacement during bending test (Fig. 15). As expected, stiffness increased with the increase of HNTs content. For small deflections the increments of stiffness were of $9.1,11.8$ and $55.1 \%$ for 3,6 and 30 $\%$ wt HNT.

\subsection{Flame Resistance}

In order to evaluate the flame resistance R, E2I and E4I were subjected to UL94 test. All of them passed the 10 second-first application without burning the cotton or reaching the fastening clip. After 10 seconds the second application all of them burned the cotton but did not reach the fastening clip. The analysis of residual flame duration showed it decreased with nanotubes increasing content. Averaged times for 10 trials are 5.12, 2,65 and $2.48 \mathrm{~s}$ for R, E2I and E4I respectively. Also, the analysis of residual incandescency duration showed the same tendency with durations of 7.74, 7.55 and $5.68 \mathrm{~s}$ for R, E2I and E4I test parts. All nanocomposites were UL94 V2 classified presenting improved flame resistance with increasing weight content of HNTs.

\section{CONCLUSIONS}

A full characterization of the rheological and mechanical behaviour of nanocomposites of polymeric matrix PA 6 based on halloysite nanotubes has been made. All results have been obtained with scaled industry equipment and ensure the feasibility of further development projects.

As the equipment used for injection molding was industry scaled to process large amounts of raw material, a lack of quality and homogeneity was detected. Against the generalized idea all masterbatches are ready-to-use in injection molding process, this research stresses the need to achieve the best dispersion prior the application in injection molding process.

XRD, FTIR, SEM and TEM techniques used for physicochemical analysis have been shown to be the most useful techniques to study the proper dispersion of nanotubes in the matrix.

The physicochemical characterization revealed that the combination of extrusion-compunding process with injection moulding gave benefits in the mechanical properties of test specimens produced. These benefits were due to the improved dispersion of nanotubes at high extrusion speeds. The direct injection of masterbatch or a low extrusion speed during mixing led to a lack of uniformity and improper dispersion of nanotubes. This conclusion has been endorsed also by the detection of the maximum values of polyamide $\gamma$ phase for E1-E3 samples.

Procesability of nanocomposites was demonstrated also with a high content of nanotubes because the measured viscosity remained in the same order of magnitude of raw PA 6 material, so no further difficulties than processing raw materials appeared.

Flame resistance was slightly improved by the use of nanotubes remaining UL94 V2 class.

The product stiffness increased with nanotubes content in all cases. The relevance of this finding is that it measures the response of the part as a whole rather than locally, as the rest of the shown trials do.

All these conclusions have been extracted from the micro and macro developed tests.

\section{REFERENCES}

[1] Lecouvet, B., Gutierrez, J.G., Sclavons, M., Bailly, C. (2011). Structure-property relationships in polyamide 12/halloysite nanotube nanocomposites. Polymer degradation and stability $A$, vol. 96, no. 2, p. 226-235.

[2] Naturalnano, Inc., from http://www. nanturalnano.com accessed on 2012-03-04.

[3] Ulrich, A., Hedicke, K. (2010). Composites of polyamide 6 and silicate nanotubes of the mineral halloysite: Influence of molecular weight on properties. Polymer, vol. 51, p. 2690-2699, DOI:10.1016/j. polymer.2010.04.041.

[4] Deliang, L., Quing, L. (2009). Correlation between interfacial interactions and mechanical properties of PA-6 doped with surface-capped nano-silica. Applied Surface Science, vol. 255, p- 2871-2877.

[5] Ismail, H., Pasbakhsh, P. (2008). Morphological thermal and tensile properties of halloysite nanotubes filled EPDM nanocomposites. Polymer Testing, vol. 27, p. 841-850, DOI:10.1016/j.polymertesting.2008.06.007.

[6] Pasbakhsh, P. (2010). EPDM/modified halloysite nanocomposites. Applied Clay Science, vol. 48, no. 3, p. 405-413, DOI:10.1016/j.clay.2010.01.015. 
[7] Hedicke-Hochstotter, K., Teck Lim, G. (2008). Novel polyamide nanocomposites based on silicate nanotubes of the mineral halloysite. Composites Science and Technology, vol. 69, no. 3-4, p. 330-334, DOI:10.1016/j. compscitech.2008.10.011.

[8] Baochun, G., Quanliang. Z. (2009). Crystallization behavior of polyamide 6/ halloysite nanotubes nanocomposites. Thermochimica Acta A, vol. 484, no. 1-2, p. 48-56, DOI:10.1016/j.tca.2008.12.003.

[9] Baochun, G., Quanliang, Z., Yanda, L. (2009). Structure and performance of polyamide 6 / halloysite nanotubes nanocm. Polymer Journal A, vol. 41, no. 10, p. 835842, DOI:10.1295/polymj.PJ2009110.

[10] Prashantha, K., Schmitt, H., Lacrampe, M.F. (2011). Mechanical behaviour and essential work of fracture of halloysite nanotubes filled polyamide 6 nanocomposites. Composites Science and Technology $A$, vol. 71 , no. 16 , p. $1859-1866$.

[11] Marney, D.C., Russell, L.J., Wu, D.Y. (2008). The suitability of halloysite nanotubes as a fire retardant for PA 6. Polymer degradation and stability, vol. 93, no. 10, p. 1971-1978, DOI:10.1016/j. polymdegradstab.2008.06.018. 